\title{
Composite Higgs bosons
}

\author{
Gabriele Ferretti** \\ Department of Physics, \\ Chalmers University of Technology, \\ Fysikgården 1, 41296 Göteborg, Sweden \\ E-mail: ferretti@chalmers.se
}

\begin{abstract}
I discuss work done in the last few years, together with my collaborators, in classifying, constructing and studying models of partial compositeness based on underlying 4D gauge theories with fermionic matter. The main motivation is trying to solve the hierarchy problem of the standard model. I start with a brief overview of the main ideas, present one particular example based on a $S U(4)$ gauge group and then discuss more recent work on the dynamics of some neutral pseudo-Nambu-Goldstone bosons that are ubiquitous in all of these models and that could be a target for coming LHC runs. I conclude with some general remarks and future directions.
\end{abstract}

Prospects for Charged Higgs Discovery at Colliders - CHARGED2018

25-28 September 2018

Uppsala, Sweden

\footnotetext{
* Speaker.

${ }^{\dagger}$ Partially supported by the The Knut and Alice Wallenberg Foundation (grant 2017.0100) and The Lars Hierta Memorial Foundation.
} 


\section{Overview}

One of the few dynamical principles available to explain the lightness of the Higgs boson is spontaneous symmetry breaking, in which the Higgs arises as a (pseudo)-Nambu-Goldstone boson (pNGB). This approach was pioneered by Kaplan and Georgi in [1] and avoids the most glaring problems of technicolor by decoupling the EW scale from the confining scale.

The approach uses the fact that a true NGB field is invariant under shift symmetry and thus cannot develop a potential. This symmetry is only approximate for the Higgs field because it is explicitly broken by the coupling to the SM. A small potential, giving rise to the Higgs vev and mass, is thus induced.

From the work of Callan, Coleman, Wess and Zumino [2, 3], we know how to parameterize the most general Effective Field Theory (EFT) describing this phenomenon. Given a global symmetry $G$ broken to a subgroup $H$ one can systematically construct the pNGB lagrangian where $G$ is realized non-linearly and then use spurions to describe the couplings to the SM.

The simplest mode [4] giving rise to (only) the Higgs doublet and preserving custodial symmetry is based on $G / H=S O(5) / S O(4)$ and the vast majority of the literature on the subject focuses on this case. It is important to keep in mind, however, that all other cosets necessarily give rise to additional pNGBs.

In fact, if one tries to realize this mechanism with a strongly coupled 4D gauge theory (hypercolor group $G_{\mathrm{HC}}$ ) with massless fermions, the three "basic" cosets are: (using Weyl notation)

\begin{tabular}{|c|c|}
\hline $4\left(\psi_{\alpha}, \tilde{\psi}_{\alpha}\right)$ Complex & $S U(4) \times S U(4)^{\prime} / S U(4)_{D}$ \\
\hline $4 \psi_{\alpha}$ Pseudoreal & $S U(4) / S p(4) \equiv S O(6) / S O(5)$ \\
\hline $5 \psi_{\alpha}$ Real & $S U(5) / S O(5)$ \\
\hline
\end{tabular}

with a pNGB content under $S U(2)_{L} \times S U(2)_{R}$ : (the Higgs field being the bi-doublet)

- Ad of $S U(4)_{D} \rightarrow(\mathbf{3}, \mathbf{1})+(\mathbf{1}, \mathbf{3})+2 \times(\mathbf{2}, \mathbf{2})+(\mathbf{1}, \mathbf{1})$

- $\mathbf{A}_{2}$ of $S p(4) \rightarrow(\mathbf{2}, \mathbf{2})+(\mathbf{1}, \mathbf{1})$

- $\mathbf{S}_{2}$ of $S O(5) \rightarrow(\mathbf{3}, \mathbf{3})+(\mathbf{2}, \mathbf{2})+(\mathbf{1}, \mathbf{1})$

This well know fact, by itself, gives little information of the possible choice of hypercolor group and representation. However, if we make the further assumption of partial compositeness [5], namely that the mass of the top quark is generated by the mixing with a Vector-Like Quark (VLQ), we can try realizing such VLQs also as a fermionic bound state of the above gauge theory. This requires the introduction of an additional set of fermions since the VLQ must carry color as well as EW quantum numbers. Denoting the new fermionic d.o.f. by $\chi$, the bound states can be schematically of type $\psi \chi \psi$ or $\chi \psi \chi$, (see [6] for details and [7] for an early model).

This requirement puts more constraints on the gauge and matter content of the theory and, with some additional assumptions, one can narrow it down to a handful of models [8,9]. The interest of these models resides mainly in the fact that many of the couplings of the effective field theory are computable from the underlying UV theory. 

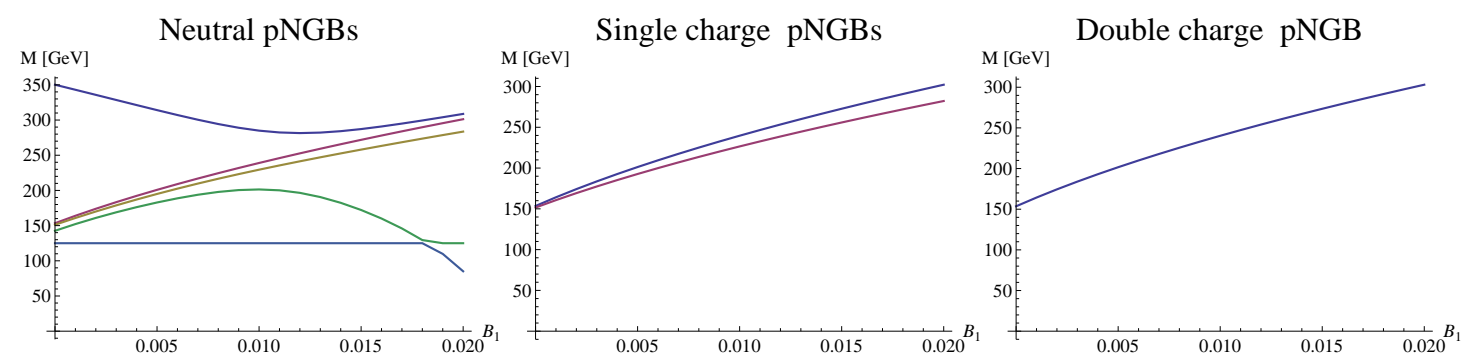

Figure 1: pNGB mass spectrum for neutral (left), charged (middle) and doubly charged (right) pNGBs for a particular choice of spurions [8], as one low energy coefficient (LEC) in the potential is varied, keeping $m_{h}=125 \mathrm{GeV}$ and $v=246 \mathrm{GeV}$. Of course, such LEC is fixed by the strong dynamics and can in principle be computed on the lattice. Picture taken from [8].
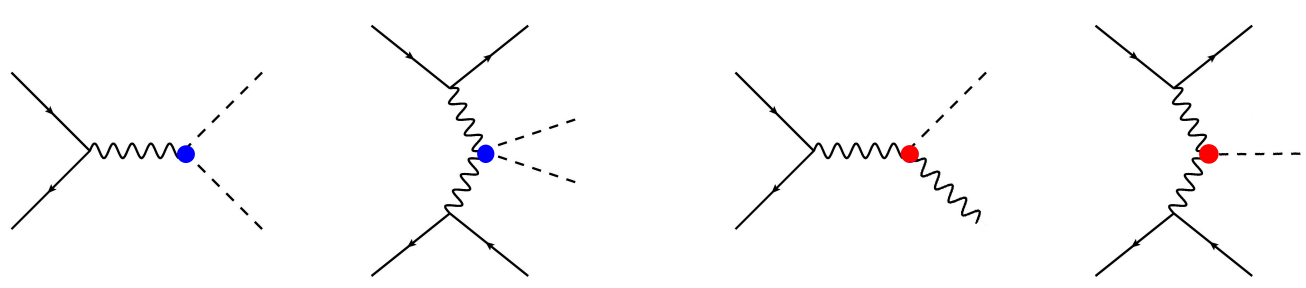

Figure 2: Double and single production modes of the charged pNGBs. The vertices in blue are the usual renormalizable EW couplings, the ones in red are the anomalous ones, suppressed by the pNGB decay constant. Picture taken from [8].

\section{Example with $S U(4)$ gauge group.}

As a concrete example, I present the model [10] based on a Hypercolor group $G_{\mathrm{HC}}=S U(4)$ with matter content $5 \times \psi \in \mathbf{6}$ (a real representation) and $3 \times(\chi, \tilde{\chi}) \in(\mathbf{4}, \overline{\mathbf{4}})$ (a complex representation).

The fermions $\psi$ are charged under the EW symmetry and their condensate generates the coset: $S U(5) / S O(5)$ à la [11], but in this case with misalignment driven by the top quark and not by an additional $U(1)$ gauge group. The fermions $\chi$ carry ordinary color and their condensate gives rise to a color octet pNGB. In this model the VLQs arise as $\chi \psi \chi$ and $\tilde{\chi} \psi \tilde{\chi}$ composite objects.

In the spectrum of Fig. 1, derived in [8] for a particular choice of spurions, one recognizes the custodial 5 (neutral, charged and doubly-charged bosons degenerate in mass) and the custodial $\mathbf{3}$ (degenerate neutral and charged) pNGBs.

The pair production of the charged members of the multiplet is almost model independent and mass limits have been recently set by ATLAS [12] to roughly $200 \mathrm{GeV}$. The single production occurs through the anomalous coupling with the EW gauge bosons and its cross section is quite suppressed. The anomaly also drives the decay of these pNGBs. Note that in the current model we would expect $\operatorname{BR}\left(H^{ \pm \pm} \rightarrow W^{ \pm} W^{ \pm}\right) \approx 100 \%$. 


\section{Additional pNGBs}

Given the low production cross sections of the charged bosons of the previous section, at LHC we might have a better shot looking at those pNGBs that couple to gluons.

There are color-carrying pNGBs arising from the condensation of the (color-carrying) fermionic fields $\chi$. These are interesting targets at LHC and their masses are expected to be well above $1 \mathrm{TeV}$, due to the one loop contribution from the gluon.

Here we focus instead on two additional pseudo-scalars that are neither charged nor colored, but still couple to gluons via an anomalous term and are ubiquitous in these models (not just the $S U$ (4) model of the previous section): $a$ and $\eta^{\prime}$. These pseudo-scalars are related to the two global $U(1)$ symmetries rotating all $\psi \rightarrow e^{i \alpha} \psi$ or all $\chi \rightarrow e^{i \beta} \chi$. Their production cross-section is much larger that the EW pNGBs in Fig. 1 due to the anomalous coupling to gluons induced by the $\chi$ s.

The linear combination free of $U(1) G_{\mathrm{HC}} G_{\mathrm{HC}}$ anomalies is associated to $a$ (light), the orthogonal one to $\eta^{\prime}$ (heavy). Importantly, the pNGB $a$ could be in the $10-100 \mathrm{GeV}$ range, where exclusions are much weaker. Its production and decay are governed by the anomaly and by the coupling to the heavy SM-fermions

$$
\begin{aligned}
\mathscr{L} & =\frac{g_{s}^{2} K_{g}}{16 \pi^{2} f_{a}} a G_{\mu \nu}^{A} \tilde{G}^{A \mu \nu}+\frac{g^{\prime 2} K_{B}}{16 \pi^{2} f_{a}} a B_{\mu \nu} \tilde{B}^{\mu v}+\frac{g^{2} K_{W}}{16 \pi^{2} f_{a}} a W_{\mu \nu}^{i} \tilde{W}^{i \mu \nu} \\
& +i C_{b} \frac{m_{b}}{f_{a}} a \bar{b} \gamma^{5} b+i C_{t} \frac{m_{t}}{f_{a}} a \bar{\tau} \gamma^{5} t+i C_{\tau} \frac{m_{\tau}}{f_{a}} a \bar{\tau} \gamma^{5} \tau .
\end{aligned}
$$

The mass $m_{a}$ and the decay constant $f_{a}$ are free parameters, but the coefficients $K_{V}$ and $C_{f}$ are computable from the quantum numbers of the hyperfermions and of the spurions and can be found in [13]. For instance, assuming $m_{a}<2 m_{t}$ and integrating out the top one finds $K_{g} \rightarrow K_{g}^{\text {eff. }}=-4.9$ and $C_{\tau}=1.5$ for the $S U(4)$ model.

It is important to stress that a pNGB $a$ with a mass less then $100 \mathrm{GeV}$ is not excluded by LEP since its coupling to the $Z$ and $W$ are much smaller than those of a Higgs boson of the same mass and thus is not produced by "alp-strahlung" or "vector boson fusion". On the other hand, LHC has a much larger production cross-section via gluon fusion (see Fig. 3).

In [13] we estimated the reach at LHC and its high luminosity upgrade in the decay channel $a \rightarrow(\tau \rightarrow e v \bar{v})(\tau \rightarrow \mu \nu \bar{v})$ after $300 \mathrm{fb}^{-1}$ and $3 \mathrm{ab}^{-1}$. This is shown in Fig. 4 for the $S U(4)$ model of Section 3. The opposite-flavor di-lepton channel suffers from the low branching ratio but has the advantage of being clean. Surely the analysis can be improved by also considering the hadronic $\tau$ decays, where the central issue becomes an adequate estimate of the systematic uncertainty.

To be able to trigger on the signal, we required the $a$ boson to be produced boosted by an ISR jet. In this, we were mostly limited by MC statistics and could not exceed $p_{T j}^{\min }=150 \mathrm{GeV}$, but we expect that raising the cut would improve the $S / B$ ratio. For trigger we used the $p_{T}$ of the muon arising from the boosted $\tau$, which we set at a minimum value of $50 \mathrm{GeV}$.

The most crucial kinematic variable in the analysis is the invariant separation $\Delta R_{e \mu}$ between the two leptons. (We still keep the isolation requirement between lepton and jet $\Delta R_{l j}>0.5$ ). Figure 5 shows the distribution of this kinematic variable for two values of the mass and the leading backgrounds. One can see that an enhancement of the $S / B$ ratio can be accomplished by cutting at a maximum of $\Delta R_{e \mu}^{\max }=1$ and setting $\Delta R_{e \mu}^{\min }$ as low as possible. Additional cuts used in the simulation are $p_{T e}>10 \mathrm{GeV}$ and $m_{e \mu}<100 \mathrm{GeV}$. 


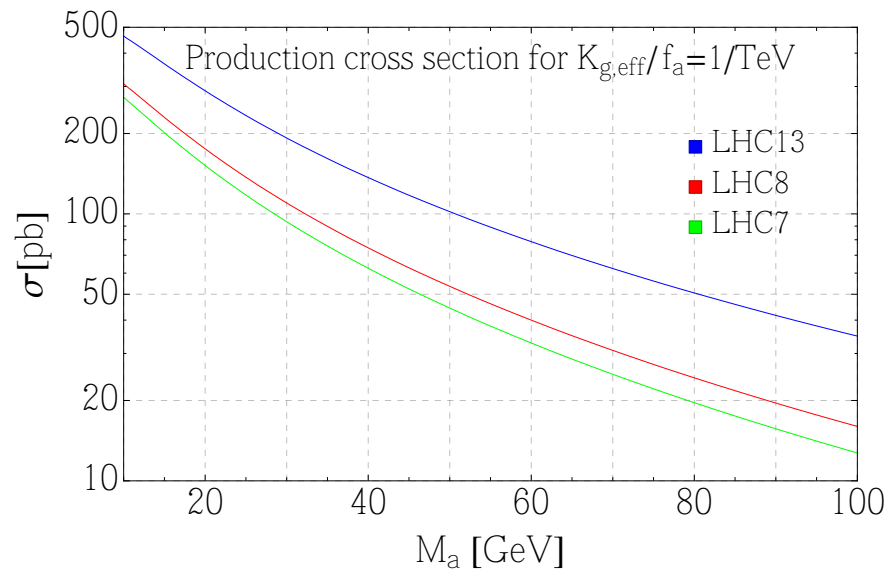

Figure 3: Gluon fusion production cross section for $a$ at LHC. The cross section appropriate for each model is obtained by rescaling by $K_{\text {eff. }}(\mathrm{TeV} / f)^{2}$. Picture taken from [13].

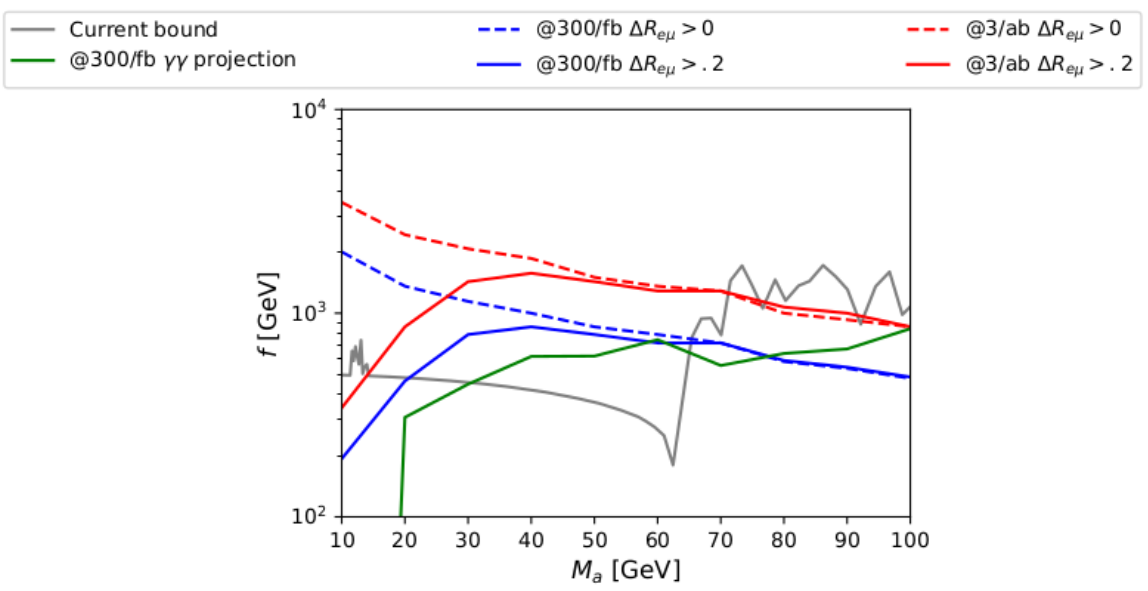

Figure 4: Expected reach for the $S U(4)$ model in the $\mu e$ channel from [13]. We also show in green the reach in the $a \rightarrow \gamma \gamma$ channel after $300 \mathrm{fb}^{-1}$ using [14].

Fig. 6 (to appear also in the CERN Yellow report on HL/HE LHC), shows the heat map plot of the current exclusion bounds for the $S U(4)$ model. In some cases the heavy $\eta^{\prime}$ gives the strongest bound. Many searches from both ATLAS and CMS are used to construct Fig. 6, too many to be all listed in this short report. Some of the most significant ones are $[15,16,17,18,19,20]$

\section{Conclusions}

I would like to summarize the discussion by collecting the main take-home points in bullet form. Some of these points have a broader applicability than the class of models at hand, an indeed they have been discussed in many other places.

- All composite Higgs models other than minimal $S O(5) / S O(4)$ contain additional pNGBs. In 


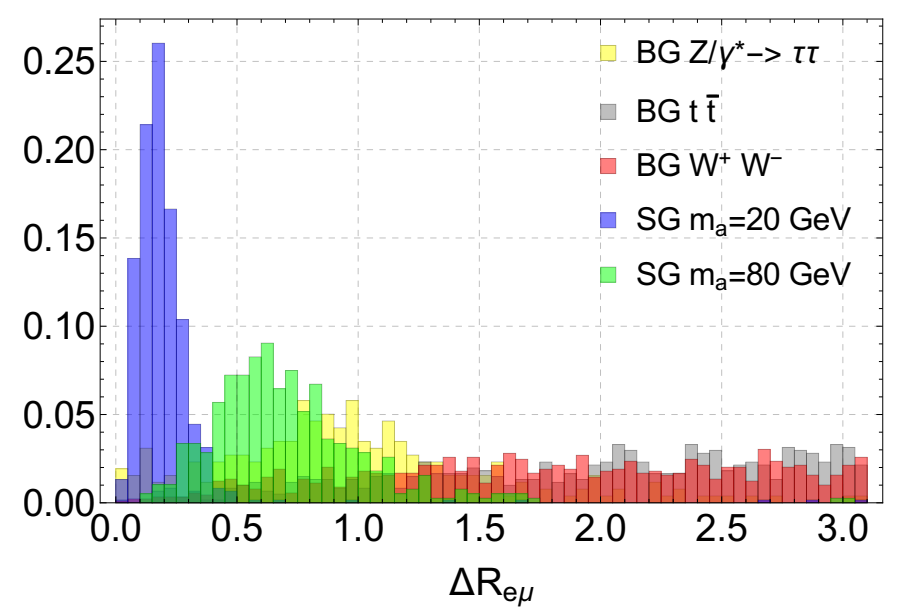

Figure 5: Angular separation $\left(\Delta R_{\mu e}\right)$ between the electron and the muon from the two decaying taus. We show the distribution for two signal (SG) for $a$ mass of $20 \mathrm{GeV}$ and $80 \mathrm{GeV}$ and compare it to the most relevant backgrounds (BG). We see that small separation angles can be a good discriminant particularly for low masses. Picture taken from [13].

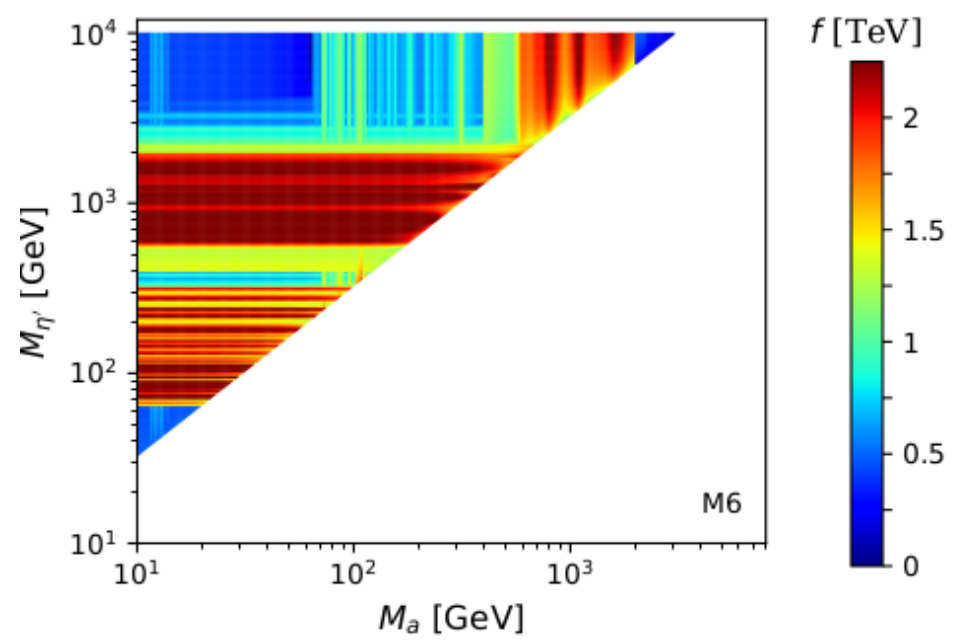

Figure 6: Exclusion region in terms of the Higgs decay constant $f$ for the $G_{\mathrm{HC}}=S U(4)$ model. The two axis represent the masses of the $a$ and $\eta^{\prime}$ respectively. The white area is not allowed.

particular, in the case of four-dimensional underlying gauge theories, one expects as minimal cosets $S U(4) / S p(4), S U(5) / S O(5)$ or $S U(4) \times S U(4)^{\prime} / S U(4)_{D}$ with the pNGB content described in the text.

- Such additional pNGBs are becoming more attractive as a chance of seeing some on-shell new physics at LHC, after many other searches have yielded negative result. This can actually be said about any weakly coupled light degree of freedom and it is one of the main motivations for the upcoming High-Luminosity LHC run, together with more precise SM 
measurements.

- Realizing models of partial compositeness via ordinary 4D gauge theories with fermionic matter provides a concrete realization where some of the LEC can be computed on a lattice. This can be compared to the situation in holography where the Higgs potential was computable perturbatively.

In this contribution I presented the basics of such constructions focusing on one example based on a $S U(4)$ hypercolor group. The EW pNGB spectrum is that of [11] with an additional neutral singlet. One interesting aspect of this model is the existence of both single and double charged scalars.

In addition to the EW sector, the $S U(4)$ model can be used as a prototype to study the dynamics of other degrees of freedom arising in these constructions. Here I focused on additional neutral pNGBs, ubiquitous in all of these models, arising from the breaking of $U(1)$ chiral symmetries. In contrast to the EW pNGBs, these scalars have larger production cross-sections via gluon-fusion and can be probed, e.g. for low masses, in the di-tau channel. I also presented a summary of current bounds and the expected reach at LHC, including the High-Luminosity run.

\section{Acknowledgments}

It's a pleasure to thank my co-authors A. Belyaev, G. Cacciapaglia, H. Cai, T. Flacke, D. Karateev, A. Parolini and H. Serodio for collaborating on various aspects of this investigation.

\section{References}

[1] D. B. Kaplan and H. Georgi, Phys. Lett. B 136 (1984) 183.

[2] S. R. Coleman, J. Wess and B. Zumino, Phys. Rev. 177 (1969) 2239.

[3] C. G. Callan, Jr., S. R. Coleman, J. Wess and B. Zumino, Phys. Rev. 177 (1969) 2247.

[4] K. Agashe, R. Contino and A. Pomarol, Nucl. Phys. B 719 (2005) 165 doi:10.1016/j.nuclphysb.2005.04.035 [hep-ph/0412089].

[5] D. B. Kaplan, Nucl. Phys. B 365 (1991) 259.

[6] G. Ferretti and D. Karateev, JHEP 1403 (2014) 077 doi:10.1007/JHEP03(2014)077 [arXiv:1312.5330 [hep-ph]].

[7] J. Barnard, T. Gherghetta and T. S. Ray, JHEP 1402 (2014) 002 doi:10.1007/JHEP02(2014)002 [arXiv:1311.6562 [hep-ph]].

[8] G. Ferretti, JHEP 1606 (2016) 107 doi:10.1007/JHEP06(2016)107 [arXiv:1604.06467 [hep-ph]].

[9] A. Belyaev, G. Cacciapaglia, H. Cai, G. Ferretti, T. Flacke, A. Parolini and H. Serodio, JHEP 1701 (2017) 094 Erratum: [JHEP 1712 (2017) 088] doi:10.1007/JHEP01(2017)094, 10.1007/JHEP12(2017)088 [arXiv:1610.06591 [hep-ph]].

[10] G. Ferretti, JHEP 1406 (2014) 142 doi:10.1007/JHEP06(2014)142 [arXiv:1404.7137 [hep-ph]].

[11] M. J. Dugan, H. Georgi and D. B. Kaplan, Nucl. Phys. B 254 (1985) 299.

[12] M. Aaboud et al. [ATLAS Collaboration], [arXiv:1808.01899 [hep-ex]]. 
[13] G. Cacciapaglia, G. Ferretti, T. Flacke and H. Serodio, Eur. Phys. J. C 78 (2018) no.9, 724 doi:10.1140/epjc/s10052-018-6183-4 [arXiv:1710.11142 [hep-ph]].

[14] A. Mariotti, D. Redigolo, F. Sala and K. Tobioka, Phys. Lett. B 783 (2018) 13 doi:10.1016/j.physletb.2018.06.039 [arXiv:1710.01743 [hep-ph]].

[15] M. Aaboud et al. [ATLAS Collaboration], Phys. Lett. B 775 (2017) 105 doi:10.1016/j.physletb.2017.10.039 [arXiv:1707.04147 [hep-ex]].

[16] V. Khachatryan et al. [CMS Collaboration], Phys. Lett. B 767 (2017) 147 doi:10.1016/j.physletb.2017.01.027 [arXiv:1609.02507 [hep-ex]].

[17] V. Khachatryan et al. [CMS Collaboration], Phys. Rev. Lett. 117 (2016) no.5, 051802 doi:10.1103/PhysRevLett.117.051802 [arXiv:1606.04093 [hep-ex]].

[18] A. M. Sirunyan et al. [CMS Collaboration], JHEP 1809 (2018) 148 doi:10.1007/JHEP09(2018)148 [arXiv:1712.03143 [hep-ex]].

[19] V. Khachatryan et al. [CMS Collaboration], JHEP 1701 (2017) 076 doi:10.1007/JHEP01(2017)076 [arXiv:1610.02960 [hep-ex]].

[20] M. Aaboud et al. [ATLAS Collaboration], JHEP 1710 (2017) 112 doi:10.1007/JHEP10(2017)112 [arXiv:1708.00212 [hep-ex]]. 